Rolf Sethe *)

\title{
Die Pflicht zum Risikomanagement im Gesellschafts-, Konzern- und Bankaufsichtsrecht
}

Der nachfolgende Beitrag untersucht am Beispiel der in Irland angesiedelten, aber für deutsche Kreditinstitute tätigen Tochter-und Zweckgesellschaften die Frage, welche Vorgaben zum Risikomanagement das Gesellschafts-, Konzern- und Bankaufsichtsrecht enthalten.

\section{Inhaltsübersicht}

I. Einleitung

II. Ausgangsfälle

1. Die Sachsen LB

2. IKB Deutsche Industriebank AG

3. Hypo Real Estate Holding AG

III. Pflicht zum Risikomanagement

1. Ebene der Einzelgesellschaft

1.1 Gesellschaftsrecht

1.2 Aufsichtsrecht

1.3 Rückwirkungen des Aufsichts- auf das Gesellschaftsrecht?

2. Konzernebene

2.1 Konzernrecht

2.2 Aufsichtsrecht

3. Die Pflicht zum Risikomanagement in den Ausgangsfällen

IV. Fazit

\section{Einleitung}

Die nachfolgenden Ausführungen konzentrieren sich auf die Frage, ob und inwieweit das Aufsichtsrecht ein an der Spitze eines Konzerns stehendes Institut zu einem konzernweiten Risikomanagement verpflichtet. Dieses Thema bewegt sich naturgemäß an der Schnittstelle zum Gesellschafts- und speziell zum Konzernrecht, denn das aufsichtsrechtlich Gebotene muss auch gesellschaftsrechtlich durchsetzbar sein. Das Risikomanagement innerhalb einer Einzelaktiengesellschaft hat Herr Dr. Krekeler im Hauptreferat bereits dargestellt, so dass die folgenden Ausführungen daran anknüpfen und - entsprechend der Zielsetzung eines Korreferats knapp - die zusätzlichen konzern- sowie die aufsichtsrechtlichen Aspekte ansprechen.

\section{Ausgangsfälle}

Über die Ursachen und die Entwicklung der Finanzkrise existieren zahlreiche ausführliche Darstellungen. ${ }^{1}$ An dieser Stelle

*) Dr. iur., LL.M. (London), Universitätsprofessor, Inhaber des Lehrstubls für Privat-, Handels- und Wirtschaftsrecht an der Universität Zürich. Bei dem Beitrag handelt es sich um die mit Belegstellen versebene Fassung seines Korreferats zum Vortrag von Dr. Hans-Dirk Krekeler (ZBB 2012, 351, in diesem Heft), das der Autor im Rabmen der Tagung „Banken in der Krise - Herausforderungen an Recht und Ökonomik"am 27. 4. 2012 an der Universität Konstanz gehalten hat. genügt ein kurzer Hinweis auf besonders markante Vorgänge, die Anlass sind, über das konzernweite Risikomanagement nachzudenken. Auffällig ist, dass zahlreiche deutsche Banken in die Krise gezogen wurden, weil sie für in Irland angesiedelte Special Purpose Vehicles (SPV) oder Tochtergesellschaften finanziell einstehen mussten. Dass diese Entwicklung die Wirtschaftsprüfer und vor allem die Aufsichtsbehörden überraschte, lag vor allem daran, dass es sich um Geschäfte handelte, die nicht bilanziert wurden und/oder die bewusst so konstruiert worden waren, dass sie nicht bilanziert werden mussten. ${ }^{2)}$

\section{Die Sachsen LB}

Die Sachsen LB und ihre Dubliner Tochterbank Sachsen LB Europe plc. (SLBE) waren vor allem im so genannten Kreditersatzgeschäft aus verbrieften Darlehen und anderen synthetischen Anlageprodukten ohne direkte Beziehung zum Kreditnehmer engagiert. ${ }^{3)}$ Sie hielten diese Anlagen entweder selbst oder über Zweckgesellschaften, namentlich die Georges Quay Funding I Limited (seit 2003), die Ormond Quay Funding plc. (seit 2004) sowie die SIV Sachsen Funding I Limited (seit 2007). Die Zweckgesellschaften waren keine Tochtergesellschaften der Bank, sondern wurden von dritter Seite gegründet und von der Bank lediglich zur Geschäftsabwicklung genutzt. Da sie keine echten Tochtergesellschaften darstellten, tauchten sie nicht in einer konsolidierten Bilanz auf. ${ }^{4)}$ Die Refinanzierung der langfristigen Anlageprodukte erfolgte über die Ausgabe von Wertpapieren mit kürzeren Laufzeiten (Ormond Quay, Sachsen Funding) und über Pensionsgeschäfte (Georges Quay). Zur Absicherung und Überbrückung stellte die Bank Liquiditätsfazilitäten zur Verfügung, die eine Laufzeit von unter 365 Tagen hatten, da sie nach den seinerzeit maßgeblichen Vorgaben von Basel I dann nicht mit Eigenkapital zu unterlegen waren. ${ }^{5)}$

Das außerbilanzielle Geschäft in den Zweckgesellschaften nahm zwischen 2003 und 2007 von vier auf 26 Mrd. € zu. Hinzu kamen bilanzierte strukturierte Produkte von $13 \mathrm{Mrd}$. $€$. Für die Bank besonders gefährlich war das Engagement in dem Con-

1) Ausführlich und sehr anschaulich zur Entstehung der Krise etwa Schröder, Handbuch Kapitalmarktstrafrecht, 2. Aufl., 2010, Rz. 1080 ff.; Rudolph, ZGR 2010, 1. Speziell aus Sicht der Schweiz Sethe, ZBB 2011, 106.

2) Als Folge der Krise wurde die Konsolidierungspflicht nach HGB durch das BilMoG v. 25. 5. 2009, BGBl I, 1102, auf Zweckgesellschaften erweitert, vgl. § 290 Abs. 2 Nr. 4 HGB. Dadurch unterfallen sie auch dem Begriff der Tochtergesellschaft in $\S 1$ Abs. 7 Satz 1 KWG und können daher im Rahmen von $\S 10$ a Abs. 1 Satz 2 KWG als nachgeordnete Unternehmen zu berücksichtigen sein.

3) Hierzu und zum Folgenden FAZ.net v. 11. 3. 2008 sowie Bericht von Ernst \& Young zur Krise der Sachsen LB v. 11. 3. 2008, www.medienservice.sachsen.de/ medien/assets/download/88646.

4) Zu Einzelheiten Kümpel/Piel, DStR 2009, 1223.

5) Sachverständigenrat, Jahresgutachten $2007 / 08$, S. 128; Marelja, Bilanzielle Grundlagen der Eigenmittelunterlegung von Risiken aus Verbriefungen, in: Becker/Marelja/König, Die Bilanzierung und Eigenmittelunterlegung von Kreditrisiken bei Kredit-Arbitrage-Programmen unter Einschaltung von Zweckgesellschaften, 2008, http://tuprints.ulb.tu-darmstadt.de/1218, S. 147; kritisch Forkel, BKR 2008, 183. Kritisch auch Marelja, ebd., S. 181, die zu dem Ergebnis kommt, dass nach damaligem Recht zumindest die Großkreditvorschriften anzuwenden gewesen wären, da eine Risikoeinheit i. S. v. § 19 Abs. 2 KWG vorlag. 
duit Ormond Quay, da sich Sachsen LB und SLBE nicht nur über Liquiditätszusagen verpflichteten, sondern eine Patronatserklärung abgaben, sämtliche wirtschaftlichen Risiken zu tragen („Valuation Agreement“). Der Bankvorstand unterließ es, das Valuation Agreement im Risikomanagementsystem zu erfassen, geschweige denn, es in den Risikoberichten oder Konzernabschlüssen zu erwähnen, und zwar selbst dann nicht, als man die Liquiditätsfazilität für Ormond Quay im Juni 2005 auf ein Volumen von bis zu $43 \mathrm{Mrd}$. $€$ erweiterte. Die Prüfgesellschaft Ernst \& Young sieht hierin auch einen Verstoß gegen Bilanzierungspflichten; die Verpflichtungen aus dem Valuation Agreement hätten seit dem Jahresabschluss 2004 im Konzernanhang veröffentlicht werden müssen. Da weder Bilanz noch Risikomanagement die riskanten Aktivitäten der Bank ausreichend widerspiegelten, war die einzige Stelle, die einen vollständigen Überblick über das Ausmaß und die Gefährlichkeit der Geschäfte hatte, der Vorstand. Da es jedoch zu mehreren Vorstandswechseln kam, waren zum Zeitpunkt des Zusammenbruchs der Bank nicht einmal auf Vorstandsebene ausreichende Kenntnisse über die Risikolage vorhanden. Ernst \& Young stellte zudem fest, dass die Bank den Liquiditäts- und Marktpreisrisiken zu wenig Bedeutung schenkte und sich stattdessen zu sehr auf Ausfallwahrscheinlichkeiten (Adressenausfallrisiken) konzentrierte. Der Bericht kommt außerdem zu dem Schluss, dass auch die Krisenbewältigung durch den Vorstand dilettantisch erfolgte.

\section{IKB Deutsche Industriebank AG}

Vergleichbar war das Geschäftsmodell der IKB, die in längerfristige forderungsbesicherte Wertpapiere (wie CDOs und ABS) investierte. Da diese Papiere in hohem Maße US-amerikanische Immobiliarkredite enthielten, schlug die Subprime-Krise auf sie durch. Die Geschäfte liefen über SPV (Rhinebridge plc., Dublin, Havenrock Ltd., Dublin, Rhineland Funding Capital Corp., Dublin). Hierbei handelte es sich um Zweckgesellschaften, die über eine Stiftung in Delaware gegründet wurden. Die IKB räumte den SPV hohe Kreditlinien ein und schöpfte über „Beraterverträge“ mit hohen Honoraren die Gewinne für sich ab, bilanzierte die SPV aber nicht als Tochtergesellschaften, da sie nicht konzernrechtlich verbunden waren. Die enormen Verdienstspannen ergaben sich auch hier aus der Fristentransformation, da man sich zur Finanzierung der höherverzinslichen langfristigen Anlagen kurzfristiger billiger Kredite bediente. Als jedoch die Kreditzinsen für kurzfristige Darlehen stiegen, versagte das Modell. Ende Juli 2007 rutschte die Bank in eine existenzbedrohende Schieflage, ${ }^{6}$ weil sie einen Liquiditätsengpass von 3,5 Mrd. € nicht decken konnte.

\section{Hypo Real Estate Holding AG}

Die Hypo Real Estate Holding übernahm 2007 im Wege eines Aktientauschs die Depfa Bank plc., Dublin. Diese stellte eine 100\%ige Tochtergesellschaft dar. Die auf Staatsfinanzierung spezialisierte Depfa Bank verfolgte ebenfalls ein Geschäftsmodell, bei dem man langfristige Kredite mittels kurzfristiger Kapitalmarktgeschäfte finanzierte. Der Umzug nach Irland wurde damit begründet, dass auf der Insel die Steuern niedrig und die
Bankenaufsicht lax seien. ${ }^{7)}$ Am 26. 9. 2008 wurde ein Liquiditätsengpass von $35 \mathrm{Mrd}$ € offenbar. ${ }^{8)}$

Bereits Anfang 2008 hatte die BaFin eine Sonderprüfung des Risikomanagements der HRE Gruppe veranlasst, die u. a. auch die irische Tochter Depfa umfasste. Der Bericht vom August 2008 listet 49 zum Teil schwere Verstöße gegen das ordnungsgemäße Risikomanagement auf. Die Bank war deshalb nicht in der Lage, alle wesentlichen Risiken zu erkennen, weil der tägliche Liquiditätsreport „nicht alle relevanten $\mathrm{Zu}$ - und Abflüsse“ umfasste und weil sie den Marktwert des größten Teils ihrer Wertpapiere und Schuldscheindarlehen nur vierteljährlich feststellte. Die internen Organisationsrichtlinien spiegelten „nicht die tatsächlichen Arbeitsabläufe wider". ${ }^{\text {") }) ~ I m ~ N a c h g a n g ~ z u ~ d e m ~}$ Bericht kam es dann noch zu einer Kontroverse über die Frage, wieweit die Kompetenzen der deutschen Aufsicht für eine laufende Prüfung über irische Tochtergesellschaften reichen. Die Bundesregierung wies darauf hin, dass bankaufsichtsrechtliche Finanzholding-Gesellschaften Finanzunternehmen im Sinne des KWG seien und daher nicht einer umfassenden, sondern nur einer eingeschränkten Aufsicht der BaFin unterlägen. Die BaFin könne bei Prüfungen im Wesentlichen nur verlangen, dass die Finanzholding-Gesellschaft Auskünfte erteilt und Unterlagen vorlegt, um die Richtigkeit der Auskünfte oder der übermittelten Daten zu überprüfen, die für die Aufsicht über das Mutterunternehmen auf konsolidierter Basis erforderlich sind. Die BaFin habe aber keine der Institutsaufsicht vergleichbaren Kompetenzen in Bezug auf die Tochtergesellschaft, was europarechtlich auch nicht vorgesehen sei. ${ }^{10)}$

\section{Pflicht zum Risikomanagement}

\section{Ebene der Einzelgesellschaft}

\subsection{Gesellschaftsrecht}

Wie Herr Dr. Krekeler herausgearbeitet hat, ist die Pflicht zum Risikomanagement Teil der Leitungspflicht des Vorstands einer AG. Bei $\S 91$ Abs. 2 AktG (Maßnahmen zur Früherkennung bestandsgefährdender Entwicklungen) handelt es sich um ein nicht abschließendes Beispiel für Risikomanagement, das keinesfalls zu einem argumentum e contrario verleiten darf. Es gehört vielmehr zur Sorgfalt eines ordentlichen Geschäftsleiters, neben möglichen bestandsgefährdenden Entwicklungen auch andere für den Unternehmenszweck bedeutende Risiken im Blick zu haben. Sein Risikomanagement muss daher alle unternehmensbezogenen, wesentlichen Risiken angemessen erfassen. ${ }^{11)}$

6) Ad-hoc-Meldung der IKB v. 30. 7. 2007, http://www.ikb.de/uploads/media/ 07_07_30_Ad-hoc-Mitteilung.pdf, die bezeichnenderweise keine Zahlenangaben enthält.

7) Frankfurter Rundschau v. 19. 8. 2009, http://www.fr-online.de/wirtschaft/falsche-geschaefte-warum-aus-der-hre-die-pleitebank-wurde,1472780,3284920.html. 8) Spiegel-Online v. 17. 8. 2009, http://www.spiegel.de/spiegel/print/d-66436856. html.

9) Der Bericht ist unveröffentlicht. Die Zitate entstammen dem Artikel von Spiegel-Online v. 27. 5. 2009, dem der Bericht vorlag, http://www.spiegel.de/ politik/deutschland/bafin-pruefbericht-wie-die-hre-in-die-katastrophe-schlitterte-a-627127.html.

10) Antwort der Bundesregierung auf die Kleine Anfrage der Abgeordneten Carl-Ludwig Thiele, Volker Wissing, Frank Schäfler, weiterer Abgeordneter und der Fraktion der FDP - Drucks. 16/10417 v. 21. 11. 2008, BT-Drucks. 16/11014, S. 8. 11) Statt vieler Pampel/Glage, in: Hauschka, Corporate Compliance, 2. Aufl., 2010, § 5 Rz. 21, 26 ff. 
Umstritten ist jedoch, ob aus dieser Sorgfaltspflicht auch eine Organisationspflicht abzuleiten ist, wonach eine Compliance-Organisation oder ein umfassendes Risikomanagementsystem (das über die Vorgaben zur Früherkennung bestandsgefährdender Entwicklungen hinausgeht) zu errichten ist. Eine solche Rechtspflicht wird unter Hinweis auf die Entstehungsgeschichte von $\S 91$ Abs. 2 AktG und § 289 HGB abgelehnt; ${ }^{12)}$ die Errichtung und der Umfang solcher Systeme stehe vielmehr im Leitungsermessen des Vorstands ${ }^{13}$ (zur Gegenauffassung sogleich unter 1.3).

\subsection{Aufsichtsrecht}

Anders ist der Befund im Bereich der Kreditinstitute und Finanzdienstleistungsinstitute, bei denen das Aufsichtsrecht eine ausdrückliche Pflicht vorsieht, Risiken angemessen zu erfassen und zu kontrollieren. So schreibt $\S 25 \mathrm{a}$ Abs. 1 Satz 1 KWG eine ordnungsgemäße Geschäftsorganisation vor. ${ }^{14)} \mathrm{Zu}$ dieser zählt nach Satz 3 der Norm ein angemessenes und wirksames Risikomanagement, auf dessen Basis ein Institut seine Risikotragfähigkeit laufend sicherzustellen hat. Die recht unübersichtliche Norm konkretisiert dann, was aufsichtsrechtlich unter einem ordnungsgemäßen Risikomanagement zu verstehen ist. Es beinhaltet zunächst die Festlegung von Strategien, Verfahren zur Ermittlung und Sicherstellung der Risikotragfähigkeit sowie die Einrichtung interner Kontrollverfahren mit einem internen Kontrollsystem und einer internen Revision. Weiterhin setzt es eine angemessene personelle und technisch-organisatorische Ausstattung des Instituts voraus, umfasst die Festlegung eines angemessenen Notfallkonzepts sowie angemessene, transparente und auf eine nachhaltige Entwicklung des Instituts ausgerichtete Vergütungssysteme für Geschäftsleiter und Mitarbeiter. Gemäß Satz 4 der Vorschrift hängt die Ausgestaltung des Risikomanagements von Art, Umfang, Komplexität und Risikogehalt der Geschäftstätigkeit ab. Seine Angemessenheit und Wirksamkeit ist vom Institut regelmäßig zu überprüfen $(\S 25 \mathrm{a}$ Abs. 1 Satz 5 KWG). Zur Ausfüllung dieser Vorgaben hat die BaFin die so genannte MaRisk ${ }^{15}$ veröffentlicht.

\subsection{Rückwirkungen des Aufsichts- auf das Gesellschafts- recht?}

Da es sich bei den im Aufsichtsrecht angesprochenen Risiken um solche aus der Tätigkeit als Bank oder Finanzdienstleistungsinstitut handelt, sind die Inhalte der Pflicht zum Risikomanagement nach Aufsichtsrecht einerseits und Gesellschaftsrecht andererseits nicht deckungsgleich, sondern es handelt sich um ein Spezialitätsverhältnis. Es wäre daher methodisch, aber auch vom Sinn und Zweck der Regelungen her verfehlt, aus den auf Spezialmaterien ausgerichteten aufsichtsrechtlichen Vorschriften der § 25a KWG, § 64a VAG, §33 WpHG, § 9 GwG eine Gesamtanalogie herzuleiten ${ }^{16)}$ und solche Systeme generell für alle Unternehmen als verpflichtend anzusehen. Auch überzeugt es nicht, aus den $\S \S 76,91,93$ AktG, $\S 130$ OWiG, § 831 BGB eine pauschale Pflicht zur Errichtung eines umfassenden Risikomanagementsystems (in der Einzelgesellschaft oder im Konzern) ableiten zu wollen, ${ }^{17)}$ denn sie schreiben lediglich ein bestimmtes Ergebnis (Wahrung der Sorgfalt) vor, nicht aber den konkreten Weg zu seiner Erreichung. Nicht alles, was Betriebswirte für sinnvoll halten, ist auch rechtlich geboten. Es muss daher dem einzelnen Vorstand überlassen bleiben, welche Maßnahmen er gemessen an Unternehmensgröße und -zweck sowie Risikoprofil ergreift; ${ }^{18)}$ im Falle von Fehlentscheidungen muss er hierfür haften. Solange also der Gesetzgeber keine allgemeine gesellschaftsrechtliche Norm zur Errichtung solcher Systeme einführt, wird man keine auf alle Unternehmen bezogene Pflicht zu einer bestimmten Organisation oder einer bestimmten Form der Risikovorsorge bejahen können. Eine solche ist auch wenig sinnvoll, denn sie würde sich auf die abstrakt-generelle Formel beschränken müssen, dass die Gesellschaften eine an Unternehmensgröße und -zweck sowie Risikoprofil ausgerichtete angemessene Organisation vorhalten müssen, und damit ist man so schlau wie zuvor. Die von der herrschenden Meinung vorgenommene Ableitung des konkreten Pflichtenumfangs in Bezug auf Compliance und Risikomanagement aus dem Leitungsermessen des Vorstands ist daher überzeugend. Dieses Ermessen umfasst auch die Frage, ob eine gesonderte Compliance-Organisation oder ein Risikomanagementsystem angesichts der Unternehmensgröße und der Art der Geschäfte erforderlich ist oder nicht.

\section{Konzernebene}

\subsection{Konzernrecht}

Betrachtet man das gesellschaftsrechtliche Schrifttum zur konzernweiten Compliance, in dessen Zusammenhang auch immer das Risikomanagement genannt wird, $\left.{ }^{19}\right)$ ist man sich einig, dass die Geschäftsleitung eines herrschenden Unternehmens für die Einhaltung der gesetzlichen Pflichten und unternehmensinternen Weisungen im Konzern zu sorgen hat. ${ }^{20)}$ Hiervon geht

12) So zur Compliance-Organisation statt vieler Koch, WM 2009, 1013 m. w. N. Zur Errichtung eines Risikomanagementsystems Fleischer, in: Spindler/Stilz, AktG, 2. Aufl., 2010, § 91 Rz. 34 f.; Hüffer, AktG, 10. Aufl., 2012, § 91 Rz. 9 - jew. m. w. N. 13) Pelz, in: Hauschka, Corporate Compliance, 2. Aufl., 2010, § 6 Rz. 1 und Gebauer/Niermann, in: Hauschka, ebd., § 36 Rz. 12 f.; Hüffer (Fußn. 12), § 91 Rz. 9. 14) Ausführlich zu Compliance-Organisation und Risikomanagement bei Instituten Eisele/Faust, in: Schimansky/Bunte/Lwowski, Bankrechts-Handbuch, 4. Aufl., 2011, § 109 Rz. 92 ff.; Fischer und Gebauer/Kleinert, in: Krieger/ Schneider, Handbuch Managerhaftung, 2. Aufl., 2010, § 19 Rz. 17 ff., § 20 Rz. 6 ff.; Gebauer/Niermann (Fußn. 13), § 36 Rz. 15 ff., 19 ff.; Gann/Rudolph, in: Hopt/ Wohlmannstetter, Handbuch Corporate Governance von Banken, 2011, S. $601 \mathrm{ff}$. 15) Mindestanforderungen an das Risikomanagement (BA) v. 20. 12. 2005, Rundschreiben 18/2005. Die aktuelle Fassung ist als Rundschreiben 11/2010 (BA) v. 15. 12.2010 veröffentlicht.

16) Eine Gesamtanalogie befürwortet U. H. Schneider, ZIP 2003, 645, 648 f.; a. A. Sethe, ZBB 2006, 243, 254 f.; Spindler, WM 2008, 905, 909.

17) Wie hier Fleischer (Fußn. 12), § 91 Rz. 34 f.; Koch, WM 2009, 1013; Hüffer (Fußn. 12), § 91 Rz. 9; Dreher, in: Festschrift Hüffer, 2010, S. 161, 162; a. A. etwa VG Frankfurt/M. WM 2004, 2157, 2160 (§ 91 Abs. 2 AktG sei deckungsgleich mit $\S$ 25a KWG); Preußner, NZG 2004, 303, 305; Säcker, NJW 2008, 3313, 3315, Fußn. 17. Siehe zur Diskussion auch Berg, AG 2007, 271, 275; Bürkle, BB 2005, 565, 568 f.; Casper, in: Bankrechtstag 2008, 139, 171; Kiethe, GmbHR 2007, 393, 396 f.; Pablke, NJW 2002, 1680, 1681; Rodewald/Unger, BB 2006, 113 f.; U. H. Schneider, ZGR 1996, 225, 230; Schwintowski, NZG 2005, 200, 201; Schwintowski/Klaue, WuW 2005, 370, 377; K. Wolf, DStR 2006, 1995 Wundenberg, Compliance und die prinzipiengeleitete Aufsicht über Bankengruppen, 2012, S. $117 \mathrm{ff}$.

18) Fleischer (Fußn. 12), §91 Rz. 34.

19) Zu diesem Zusammenhang ausführlich Pampel/Glage (Fußn. 11), § 5 Rz. 1 ff. 20) Vgl. Bürkle, BB 2007, 1797, 1799; Casper, in: Bankrechtstag 2008, 139, 170 f.; Fleischer, CCZ 2008, 1, 5; Hüffer (Fußn. 12), § 76 Rz. 9a; Immenga, in: Festschrift Schwark, 2009, S. 199, 204; Koch, WM 2009, 1013, 1015 ff.; Lösler, NZG 2005, 104, 105 ff.; Schneider/Schneider, ZIP 2007, 2061, 2064 sowie die Nachweise bei Habersack, in: Festschrift Möschel, 2011, S. 1175. 
auch Ziff. 4.1.3 des Corporate Governance Kodex aus. Die genaue Herleitung dieser Pflicht ist jedoch alles andere als geklärt und es kann insoweit auf die gerade wiedergegebene Diskussion zur Einzelgesellschaft verwiesen werden. Darüber hinaus sind folgende drei Aspekte zu erwägen:

Im deutschen Konzernrecht ist bereits die hier interessierende Ausgangsfrage, ob es eine Konzernleitungspflicht der Mutter gibt, aus der man dann auch eine Gesamtverantwortung für das Risikomanagement im Konzern ableiten könnte, umstritten. Die ganz herrschende Meinung verneint - gestützt auf $\S 311$ AktG und auf das Trennungsprinzip - eine solche Pflicht; der Vorstand der herrschenden AG ist nicht berechtigt oder gar verpflichtet, auch die Tochtergesellschaften umfassend zu leiten. ${ }^{21)}$ Eine Weisungsbefugnis besteht nach dem Aktienkonzernrecht nur ausnahmsweise, wenn es sich um eingegliederte AGs (§ 323 Abs. $1 \mathrm{AktG}$ ) oder um abhängige AGs im Vertragskonzern handelt (§§ 291, 308 AktG). ${ }^{22)}$ Allerdings wird auch hier im Schrifttum betont, dass der Konzernvorstand nur eine Weisungsbefugnis, aber keine Weisungspflicht hat. ${ }^{23)}$ Das Schrifttum geht in den Fällen des Vertragskonzerns und der eingegliederten AG zu Recht davon aus, dass der Konzernvorstand verpflichtet ist, ein System zur konzerndimensionalen Risikoerfassung und -auswertung einzurichten. Aufgrund der höheren Komplexität der Vorgänge im Konzern wird das Leitungsermessen des Vorstands regelmäßig dahingehend auszuüben sein, eine bestimmte Organisation für Compliance und Risikomanagement vorzusehen. Dieses kann er bei eingegliederten AGs und im Vertragskonzern aufgrund seines Weisungsrechts auch durchsetzen. ${ }^{24)}$

Im faktischen Konzern besteht dagegen weder eine gesetzlich verankerte Einflussmöglichkeit der Obergesellschaft noch ein unmittelbares Kontrollrecht. ${ }^{25}$ Damit aber steht die Muttergesellschaft im faktischen Konzern vor der Frage, wie sie ihre eigene Geschäftsführung ausrichten muss, um ihrer Pflicht nachzukommen, Schäden von der eigenen Gesellschaft aufgrund der Beteiligung an der Tochtergesellschaft abzuwenden. Eine direkte Inanspruchnahme der Mutter aufgrund von Fehlverhalten in Tochtergesellschaften ist in jüngster Zeit gleich mehrfach virulent geworden: (1) So hat die SEC die Siemens AG für Korruptionszahlungen im Zeitraum von 1996 bis 2007 in Anspruch genommen, die ihre argentinische Tochtergesellschaft Siemens S.A. („Siemens Argentina“) begangen hat. ${ }^{26)}$ Die Siemens AG verpflichtete sich, 350 Mio. US-\$ unrechtmäßig erzielten Gewinns herauszugeben und eine Strafe von 450 Mio. US-\$ zu bezahlen; in Deutschland wurden weitere Zahlungen an die Strafverfolgungs- und Steuerbehörden geleistet, die sich auf 395 Mio. US-\$ und 201 Mio. US-\$ belaufen haben. ${ }^{27)}$ Insgesamt dürfte der Korruptionsskandal der Tochter die Muttergesellschaft rund eine Mrd. € gekostet haben. ${ }^{28)}$ (2) Im Konzern werden von einer Tochtergesellschaft ausgehende Kartellverstöße der Mutter zugerechnet, da Mutter- und Tochtergesellschaft als wirtschaftliche Einheit (ein Unternehmen im kartellrechtlichen Sinne) behandelt werden, wenn die Mutter Einfluss auf die Geschäftsführung der Tochter hat, was bei 100\%igem Anteilsbesitz widerleglich vermutet wird. ${ }^{29)}$ (3) Neben die Fälle der Inanspruchnahme der Mutter aufgrund von zugerechnetem Fehlverhalten der Tochter treten Fälle, in denen die Haftung der Mutter aufgrund rechtsgeschäftlicher Einstandspflich- ten ausgelöst wird. So beruhte die Haftung der Sachsen LB auf dem Valuation Agreement; im Falle IKB und HRE folgte sie aus den Liquiditätszusagen für die SPV. Da der Muttergesellschaft im faktischen Konzern gerade keine Weisungsmöglichkeit zusteht, ist sie verpflichtet, ihren faktischen Einfluss bei der Tochter dahin gehend zu nutzen, dass ihr ausreichend Informationen für das Risikomanagement zur Verfügung gestellt werden. Gelingt ihr dies nicht, muss sie innerhalb der Muttergesellschaft eigene Maßnahmen zur Identifizierung der aus den Tochtergesellschaften resultierenden Risiken ergreifen und die ihr zur Verfügung stehenden Erkenntnisquellen nutzen. ${ }^{30}$

Von der soeben erörterten Gestaltung, bei der der Mutter eine Inanspruchnahme aufgrund von Zurechnungstatbeständen oder einer Verlustausgleichspflicht droht und in der deshalb ein Eigeninteresse der Mutter an einer konzernweiten Compliance und einem konzernweiten Risikomanagementsystem besteht, trennt das Schrifttum die Gestaltung, bei der keine unmittelbare Inanspruchnahme der Mutter droht und diese daher „nur“ den Wert ihres Beteiligungsbesitzes im Auge hat. Hier bestehe keine Pflicht zu einem konzernweiten Risikomanagement. ${ }^{31)}$ Dieses Unterscheidungskriterium vermag nicht zu überzeugen, denn auch der Beteiligungsbesitz kann für die Mutter so wesentlich sein, dass sein Verlust auch die Mutter existenziell trifft. Wenn also die Beteiligung für die Mutter von bedeutendem Wert ist, wird man auch hier eine Einbeziehung in das Risikomanagement verlangen müssen.

Im Ergebnis ist die Reichweite der Pflicht zum Risikomanagement im Konzern von der jeweiligen Konzernierungsform abhängig, da diese die Grenzen der Einflussmöglichkeiten der Obergesellschaft vorgibt. Der Konzernvorstand kann nicht zu etwas verpflichtet werden, was ihm rechtlich nicht gestattet ist. ${ }^{32)}$ Er ist jedoch stets gehalten, zumindest die ihm innerhalb der Muttergesellschaft zur Verfügung stehenden Erkenntnis-

21) Fleischer (Fußn. 12), § 76 Rz. 86 ff.; Mertens/Cahn, in: Kölner Komm. z. AktG, 3. Aufl., 2010, § 76 Rz. 65; Seibt, in: K. Schmidt/Lutter, AktG, 2. Aufl., 2010, § 76 Rz. 16; Hüffer (Fußn. 12), § 76 Rz. 17; Martens, in: Festschrift Heinsius, 1991 S. 523, 531; Mülbert, Aktiengesellschaft, Unternehmensgruppe und Kapitalmarkt, 2. Aufl., 1996, S. 29 ff.; a. A. Hommelhoff, Die Konzernleitungspflicht, 1982, S. 43 ff., 165 ff., 184 ff.; Timm, Die Aktiengesellschaft als Konzernspitze, 1980, S. 95 ff.; Timm, NJW 1987, 977, 980; Kropff, ZGR 1984, 112, 116.

22) In anderen als aktienrechtlichen Konzernverhältnissen kommen die jeweiligen speziellen gesellschaftsrechtlichen Regelungen zum Tragen (insbesondere das Weisungsrecht nach $\S 37$ Abs. $1 \mathrm{GmbHG}$ gegenüber einer nachgeordneten $\mathrm{GmbH}$ ), auf die aus Platzgründen nicht näher eingegangen wird.

23) Spindler, WM 2008, 905, 915.

24) Fleischer (Fußn. 12), § 76 Rz. 97; Lutter, in: Festschrift Goette, 2011, S. 289, 296.

25) Lutter (Fußn. 24), S. 289, 293 f.

26) Vgl. die Maßnahmen der SEC gegen die Siemens AG, München, http://www.scribd.com/doc/30881452/SEC-v-Siemens, sowie die Maßnahmen der SEC gegen die verantwortlichen Organmitglieder, http://www.scribd.com/ doc/75581250/SEC-Siemens-Complaint.

27) Vgl. http://www.scribd.com/doc/75581250/SEC-Siemens-Complaint, Rz. 19. 28) Welt-Online v. 13. 12. 2008, http://www.welt.de/wirtschaft/article2873135/ Siemens-zahlt-Milliarde-im-Schmiergeld-Skandal.html. Weitergehend Lutter (Fußn. 24), S. 289, 292, der von 2,5 Mrd. € ausgeht.

29) EuGH, Urt. v. 10. 9. 2009 - Rs C-97/08 P, ZIP 2010, 392, Ls. 4 - Akzo Nobel, dazu EWiR 2010, 149 (Lieder/Kliebisch); EuGH, Urt. v. 14. 12. 2006 - Rs C-217/05, Ls. 3 - Confederación Española de Empresarios de Estaciones de Servicio; BKartA - B1-200/06 - Etex.

30) Fleischer (Fußn. 12), § 76 Rz. 97; Lutter (Fußn. 24), S. 289, 296.

31) Habersack (Fußn. 20), S. 1181 ff. m. w. N. auch zur a. A.

32) Fleischer (Fußn. 12), § 92 Rz. 62; Spindler, WM 2008, 905, 916; Lutter (Fußn. 24), S. 289, 294 (weiter dagegen auf S. 297). 
Tabelle 1: Begriff der übergeordneten Unternehmen ${ }^{33)}$

\begin{tabular}{|c|c|c|}
\hline Gruppe & KWG & Übergeordnetes Unternehmen \\
\hline Institutsgruppe & $\S 10 \mathrm{a}$ Abs. 1 & Inländisches Mutterinstitut nach $\S 1$ Abs. 7a KWG ${ }^{34)}$ \\
\hline Horizontale Unternehmensgruppe & $\S 10 \mathrm{a}$ Abs. 2 & $\begin{array}{l}\text { Gruppenangehöriges Einlagenkreditinstitut oder Wertpapierhan- } \\
\text { delsunternehmen mit Sitz im Inland und höchster Bilanzsumme }\end{array}$ \\
\hline Inländische Finanzholding-Gruppe & $\S 10$ a Abs. 3 Satz 1 & $\begin{array}{l}\text { Gruppenangehöriges inländisches Einlagenkreditinstitut oder } \\
\text { Wertpapierhandelsunternehmen, das selbst keinem anderen } \\
\text { gruppenangehörigen inländischen Institut nachgeordnet ist }\end{array}$ \\
\hline Ausländische Finanzholding-Gruppe & $\S 10 \mathrm{a}$ Abs. 3 Satz 3 & $\begin{array}{l}\text { Gruppenangehöriges inländisches Einlagenkreditinstitut oder } \\
\text { Wertpapierhandelsunternehmen, das selbst keinem anderen in- } \\
\text { ländischen Institut nachgeordnet ist und mit der höchsten Bi- } \\
\text { lanzsumme im Vergleich zu jedem anderen Einlagenkreditinstitut } \\
\text { oder Wertpapierhandelsunternehmen mit Sitz in einem anderen } \\
\text { EWR-Staat }\end{array}$ \\
\hline Finanzkonglomerate & $\S 10 \mathrm{~b}$ Abs. 3 & Übergeordnetes inländisches Finanzkonglomerats-Unternehmen \\
\hline Gruppen zur Unterkonsolidierung & $\S 10 \mathrm{a}$ Abs. 14 & $\begin{array}{l}\text { Inländisches Institut bzw. übergeordnetes Unternehmen der in- } \\
\text { ländischen Finanzholding-Gruppe }\end{array}$ \\
\hline
\end{tabular}

quellen zu nutzen, um aus den Tochtergesellschaften drohende Risiken zu identifizieren. ${ }^{35)}$ Darüber hinaus muss er natürlich auch potenzielle Risiken aus anderen Quellen, wie SPV, einbeziehen. Deren Struktur ist ihm bekannt, da er selbst diese Konstrukte gewählt hatte. Im Rahmen des Risikomanagements durfte sich eine deutsche Konzernspitze also nicht auf den Standpunkt zurückziehen, die aus Irland drohenden Verbindlichkeiten seien nicht einzubeziehen, da es sich bei den SPV nicht um Tochtergesellschaften „im formellen Sinne“ handele. Gleiches gilt im Übrigen für die Einbeziehung der revolvierenden Liquiditätsfazilitäten. Dass die Liquiditätsfazilitäten damals nicht mit Eigenkapital unterlegt werden mussten, befreit nicht von ihrer Einbeziehung in das Risikomanagement.

\subsection{Aufsichtsrecht}

Besondere Regelungen finden sich zum Risikomanagement im „Finanzkonzern“, die auf den Vorgaben des Gemeinschaftsrechts beruhen. ${ }^{36)} \S 25 \mathrm{a}$ Abs. 1a, 1b KWG verpflichtet übergeordnete Unternehmen von Institutsgruppen, FinanzholdingGruppen, Instituten i. S. d. § 10a Abs. 14 KWG oder von Finanzkonglomeraten zum Risikomanagement. In einem ersten Schritt ist daher zunächst der Kreis der in die Gruppenbetrachtung einzubeziehenden Unternehmen zu ermitteln. Dieser ergibt sich aus dem aufsichtsrechtlichen Konsolidierungskreis, der wiederum in den $\S 10$ a Abs. 1 bis 3 und Abs. 14, $\S 10 \mathrm{~b}$ Abs. 3 KWG geregelt ist. Im zweiten Schritt wird das übergeordnete Unternehmen festgestellt. Der Begriff des übergeordneten Unternehmens hängt davon $\mathrm{ab}$, um welche Art der Gruppe es sich handelt, wie Tabelle $1^{37)}$ zeigt.

Die MaRisk schreibt in AT 2.1 ein konzernweites Risikomanagement vor. Das Risikomanagement auf Gruppenebene erstreckt sich auf alle wesentlichen Risiken der Gruppe. Es kommt nicht darauf an, ob die Risiken von konsolidierungspflichtigen Unternehmen selbst begründet wurden oder nicht; daher sind auch Risiken aus nicht konsolidierungspflichtigen Zweckgesell- schaften zu erfassen (MaRisk AT 4.5 Tz. 1). ${ }^{38)}$ Allerdings verweist $\S 25 a$ Abs. 1a Satz 2, Abs. 1b Satz 3 KWG auf $\S 10$ a Abs. 12 Satz 2, § 10b Abs. 6 Satz 2 KWG, wonach das übergeordnete Unternehmen zur Erfüllung dieser Pflichten nur innerhalb der vom Gesellschafts- und Konzernrecht gezogenen Grenzen tätig werden darf. ${ }^{39)}$ Wegen dieser Einschränkung schrieb die MaRisk in der Fassung von 2007 vor, dass das Risikomanagement „im Rahmen der gesellschaftsrechtlichen Möglichkeiten" zu erfolgen habe. ${ }^{40)}$ Seit der Fassung der MaRisk vom 14. 8. 2009 ist diese Einschränkung entfallen, so dass der Geltungsanspruch der MaRisk nun weiter erscheint. Allerdings kann ein Rundschreiben die gesetzlichen Vorgaben nicht aufheben.

Legt man diese Grenzen zugrunde, ergibt sich also die oben bereits entwickelte Zweiteilung: (1) Bei Vorliegen eines Beherrschungsvertrags und einer Eingliederung kann ein konzern-

33) Tabelle angelehnt an Braun/Wolfgarten, in: Boos/Fischer/Schulte-Mattler, Kreditwesengesetz, 4. Aufl., 2012, § 25a Rz. 829.

34) Zweckgesellschaften können seit der Änderung von $\S 290$ HGB unter den Tatbestand des nachgeordneten Unternehmens fallen, vgl. Fußn. 2.

35) So ausdrücklich in Bezug auf die Sachsen LB auch Lutter (Fußn. 24), S. 289, 296. 36) Die Gruppenbetrachtung wurde ursprünglich in $\S 25 \mathrm{a}$ Abs. $1 \mathrm{KWG}$ verankert durch Art. 6 Nr. 25 des Vierten Finanzmarktförderungsgesetzes (FMFG) v. 21. 6. 2002, BGB1 I 2010. Mit Art. 1 Nr. 24 lit. b des Finanzkonglomeraterichtlinien-Umsetzungsgesetzes (FKRL-UmsG) v. 21. 12. 2004, BGBI I, 3610, wurde Abs. 1a eingeführt, der die Organisationspflichten auf Institutsgruppen, Finanzholding-Gruppen und Finanzkonglomerate erstreckte. Mit dem Art. 1 Nr. 33 lit. b des Gesetzes zur Umsetzung der neu gefassten Bankenrichtlinie und der neu gefassten Kapitaladäquanzrichtlinie (BKRUG) v. 17. 11. 2006, BGB1 I, 2606, wurde die Anwendung auch auf Institute nach $\S 10$ a Abs. 14 KWG vorgesehen. Durch Art. 1 Nr. 21 des Gesetzes zur Umsetzung der Richtlinie 2010/78/EU v. 24. 11. 2010 im Hinblick auf die Errichtung des Europäischen Finanzaufsichtssystems (EUFAAnpG) v. 4. 12. 2011, BGBl I, 2427, erfolgte die heute gültige Aufsplittung in Abs. 1a und $1 \mathrm{~b}$

37) S. o. Fußn. 33.

38) Zur 2009 erfolgten Änderung des Kreises der konsolierungspflichtigen Unternehmen vgl. Fußn. 2.

39) Binder, in: Hopt/Wohlmannstetter, Handbuch Corporate Governance von Banken, 2011, S. 696, 701 ff.; Braun/Wolfgarten (Fußn. 33), § 25a Rz. 838; Langen, in: Schwennicke/Auerbach, KWG, 2009, § 25a Rz. 113; Spindler, WM 2008, 905, 916.

40) Mindestanforderungen an das Risikomanagement - MaRisk, Rundschreiben 5/2007 v. 30. 10. 2007. 
weites Risikomanagement durchgesetzt werden. (2) Bei faktischer Konzernierung ist das übergeordnete Unternehmen auf die Mitwirkung der nachgeordneten Unternehmen angewiesen. ${ }^{41)}$ Aus der Verpflichtung, ein konzernweites Risikomanagement zu gewährleisten, leitet eine Ansicht ab, das übergeordnete Unternehmen sei zumindest für den Fall, dass in einem nachgeordneten Unternehmen wesentliche Risiken schlummerten, aufsichtsrechtlich zum Abschluss eines Beherrschungsvertrags verpflichtet, um die Durchsetzbarkeit sicherzustellen. ${ }^{42}$ Dies überzeugt nicht, denn wenn das KWG schon für den Fall der sehr konkreten Eigenmittelvorschriften ausdrücklich einen Vorbehalt der Grenzen des Gesellschaftsrechts erklärt und gerade keine Pflicht zum Abschluss eines Beherrschungsvertrags kennt, kann man dies schwerlich für die viel vageren Vorgaben zum Risikomanagement behaupten.

Die BaFin kann entweder ihre Mittel nach § 2c KWG bei der Gruppenbildung nutzen oder bei schon vorhandenen Gruppen zur Durchsetzung eines konzernweiten Risikomanagements den indirekten Zwang über eine Anordnung nach $\S 45 \mathrm{~b}$ KWG, falls das nachgeordnete Unternehmen seine Mitwirkung am konzernweiten Risikomanagement verweigert. ${ }^{43)}$ So kann sie dem übergeordneten und/oder dem nachgeordneten Institut zusätzliche Eigenmittel (§ 45b Abs. 1 Satz 2 KWG), Maßnahmen zur Reduzierung von Risiken, die sich aus bestimmten Arten von Geschäften und Produkten oder der Nutzung bestimmter Systeme ergeben (§ 45b Abs. 1 Satz 1 Nr. 1 KWG), die Einführung eines Erlaubnisvorbehaltes für die Errichtung von Zweigstellen ( $§ 45 \mathrm{~b}$ Abs. 1 Satz 1 Nr. 2 KWG) sowie die Beschränkung oder das Verbot bestimmter Geschäftsarten, namentlich die Annahme von Einlagen, Geldern oder Wertpapieren und die Kreditgewährung (§ 45b Abs. 1 Satz 1 Nr. 3 KWG), aufgeben. Diese Maßnahmen können einzeln, gemeinsam oder mit Maßnahmen nach $\S 25 a$ Abs. 1 Satz 8 KWG ergriffen werden. Als ultima ratio verbleibt schließlich noch die Aufhebung der aufsichtsrechtlichen Zulassung des übergeordneten und/oder des nachgeordneten Instituts gem. § 33 Abs. 3 Satz 2 Nr. 1 und 3, § 35 Abs. 2 Nr. 3 KWG.

Die näheren Inhalte des Risikomanagements ergeben sich aus der MaRisk. Sie können aus Platzgründen hier nicht dargestellt werden. Auffällig ist jedoch, dass das Aufsichtsrecht nicht nur das „Ob“, sondern auch das „Wie“ detailliert vorgibt und damit organisatorische Pflichten enthält, die das Gesellschaftsrecht so nicht kennt (s. insbesondere MaRisk AT 4.5 Tz. 2 - 6, AT 4.3.2).

\section{Die Pflicht zum Risikomanagement in den Ausgangs- fällen}

Wendet man die soeben herausgearbeiteten Regeln an, konnte und musste die HRE als Alleinaktionärin der Depfa Bank ihren gesellschaftsrechtlichen Einfluss nutzen, um ein konzernweites Risikomanagement zu etablieren. Diese Pflicht ergab sich nicht nur aus dem Gesellschaftsrecht, sondern auch aus dem Aufsichtsrecht, da es sich um eine Institutsgruppe handelte.

Im Falle der Sachsen LB und der IKB lag dagegen kein Gruppentatbestand vor, da die irischen Zweckgesellschaften nach damaligem Recht keine nachgeordneten Unternehmen darstellten.
Daher konnten und mussten sie aufsichtsrechtlich die SPV nicht in ein konzernweites Risikomanagement einbeziehen. Dieser Umstand befreite die beiden Banken jedoch nicht von der Pflicht, auf Ebene ihres eigenen Risikomanagements die aus den Geschäften in Irland drohenden Risiken aus den Liquiditätsfazilitäten und dem Valuation Agreement einzubeziehen; entsprechende Erkenntnismöglichkeiten waren in beiden Instituten vorhanden; sie wurden nicht genutzt oder ignoriert. Die von den Instituten ins Feld geführten Argumente wie „außerbilanziell“ und „nicht konsolidierungspflichtig“ haben zwar für Bilanzfragen eine Rolle gespielt (was aus Platzgründen nicht vertieft werden $k_{a n n}{ }^{44)}$ ), dürfen aber nicht den Blick darauf verstellen, dass eklatante Verstöße im Bereich des Risikomanagements und darüber hinaus auch des $\S 91$ Abs. 2 AktG vorlagen. Bemerkenswert ist zudem der Umstand, dass die Verbriefungstransaktionen ganz überwiegend in Irland stattgefunden haben, weil hier nicht nur die Steuer tief, sondern auch die Aufsicht lax war. Die Banken haben bewusst eine Aufsichtsarbitrage ausgenutzt; offenbar wollten sie auch nicht, dass man allzu genau hinschaut.

\section{Fazit}

1. Die Pflicht zum Risikomanagement ist Teil der Leitungspflicht des Vorstands einer AG. Der Vorstand muss nach Unternehmensgröße und Art der Geschäfte angemessene Vorkehrungen treffen. Konkrete organisatorische Vorgaben schreibt das Gesetz jedoch nicht vor und solche lassen sich auch nicht im Wege von Gesamtanalogien zu Spezialvorschriften ableiten.

2. Die Notwendigkeit eines konzernweiten Risikomanagements hängt einerseits davon $a b$, ob der Mutter eine Inanspruchnahme aufgrund einer Zurechnung des Verhaltens der Tochter oder einer Haftungsübernahme oder Verlustausgleichspflicht droht, und anderseits davon, ob die Beteiligung an der Tochtergesellschaft für die Mutter von wesentlicher wirtschaftlicher Bedeutung ist.

3. Während die Durchsetzung eines konzernweiten Risikomanagementsystems im Vertragskonzern und bei eingegliederten Aktiengesellschaften unproblematisch möglich ist, setzt sie im faktischen Konzern die Mitwirkung des nachgeordneten Unternehmens voraus. Die Reichweite der Pflicht zum Risikomanagement im Konzern folgt also der jeweiligen Konzernierungsform. Der Konzernvorstand kann nicht zu etwas verpflichtet werden, was ihm rechtlich nicht gestattet ist.

4. Diese gesellschaftsrechtliche Grenze schlägt auch auf die organisatorischen Vorgaben im Bankaufsichtsrecht durch. Allerdings hält das Aufsichtsrecht eine Reihe von Sanktionen bereit, die als Druckmittel für den Fall eingesetzt werden können, dass

41) Ausführlich dazu Binder (Fußn. 39), S. 701 ff., der zu Recht darauf hinweist, dass in der Praxis die Unterschiede zwischen beiden Gestaltungen gering sein dürften (ebd., S. 708).

42) Langen (Fußn. 39), § 25a Rz. 113; a. A. Braun/Wolfgarten (Fußn. 33), § 25a Rz. 838, Fußn. 841

43) Ebenso Binder (Fußn. 39), S. 710

44) Ausführlich Becker, Die Konsolidierung von Zweckgesellschaften vor dem Hintergrund der Subprime-Krise, in: Becker/Marelja/König, Die Bilanzierung und Eigenmittelunterlegung von Kreditrisiken bei Kredit-Arbitrage-Programmen unter Einschaltung von Zweckgesellschaften, 2008, http://tuprints.ulb.tu-darmstadt.de/1218, S. $100 \mathrm{ff}$ 
nachgeordnete Unternehmen im faktischen Konzern nicht an einem konzernweiten Risikomanagement mitwirken wollen.

5. Die im Zuge der Finanzmarktkrise oft gehörte Behauptung, die deutschen Institute hätten die in Irland ansässigen Zweckgesellschaften nicht in ihr Risikomanagement einbeziehen müssen, da sie keine echten Tochtergesellschaften gewesen seien, überzeugt nicht. Das Risikomanagement erstreckt sich auch auf Risiken, die aus anderen Quellen als Tochtergesellschaften erwachsen. Die Geschäfte der Zweckgesellschaften wären daher im Risikomanagement des deutschen „Mutter“-Instituts zu berücksichtigen gewesen.

6. Im Gefolge der Finanzmarktkrise wurden zahlreiche Stellschrauben des Aufsichtsrechts neu justiert, ${ }^{45)}$ um zu verhindern, dass eine solche Krise nochmals auftritt. Man sollte sich jedoch hüten zu glauben, dass damit die Gefahr einer erneuten Krise endgültig gebannt sei, denn die beaufsichtigten Institute haben - wie gezeigt - in der Vergangenheit systematisch Regelungslücken und Schwächen des Aufsichtssystems genutzt. Gerade das Beispiel der Ansiedlung der Verbriefungsindustrie in Irland belegt dies. Man wirtschaftete nach der Devise, dass alles, was nicht ausdrücklich verboten ist, auch gemacht werden darf. Nur durch neue Regelungen, die zumeist vergangenheitsbezogene Ausbesserungen sind, wird sich diese Grundhaltung nicht ändern. Oder um es mit dem - passenderweise irischen - Schriftsteller George Bernard Shaw (1856 - 1950) zu sagen: „Wir werden nicht durch die Erinnerung an unsere Vergangenheit weise, sondern durch die Verantwortung für unsere Zukunft.“

45) Vgl. im vorliegenden Zusammenhang insbesondere oben Fußn. 2. Zudem existieren noch zahlreiche Reformvorschläge zum Risikomanagement, auf die aber aus Platzgründen hier nicht eingegangen werden kann, vgl. stattdessen Gann/Rudolph (Fußn. 14), S. $620 \mathrm{ff}$

\section{Philipp Maximilian Holle*)}

\section{Diskussionsbericht zu dem Referat von Hans-Dirk Krekeler und zu dem Korreferat von Rolf Sethe - Gesellschaftsrechtliche Aspekte des Risikomanagements}

Die Diskussion entzündete sich zunächst an der Frage, wie effektiv die innergesellschaftlichen Mechanismen zur Inanspruchnahme von Leitungsorganen sind. Grund war die Feststellung, dass bis heute kaum eine Gesellschaft den Versuch unternommen hat, ihre Leitungsorgane für mögliche Verfehlungen aus der Bankenkrise haftbar zu machen. Hierfür wurden im Wesentlichen drei Gründe herausgeschält: Erstens seien die eingetretenen Verluste namentlich im Bankensektor zumeist so exorbitant hoch, dass sie sich durch eine Inanspruchnahme der Vorstandsmitglieder nur zu einem Bruchteil wieder ausgleichen ließen. Zweitens würde ein Prozess dazu führen, dass Beweismittel offen gelegt werden, die unter Umständen auch eine Sanktionierung des Unternehmens im Außenverhältnis tragen. Man liefere externen Behörden hiermit gleichsam die Grundlage für weitere Verfahren gegen die Gesellschaft, die mit zusätzlichen, weitaus empfindlicheren Einbußen für das Gesellschaftsvermögen enden könnten. Drittens drohten massive Imageverluste. Auch wurde die Frage aufgeworfen, wie stabil die Informationsgrundlagen sind, auf deren Basis der Aufsichtsrat Ansprüche überhaupt erst prüfen kann. Krekeler (ZBB 2012, 351, in diesem Heft) führte hierzu aus, dass die Kontrollgremien heute deutlich intensiver agierten als früher. Man sei sich seiner Verantwortung bewusst und gehe „mehr ins Detail“. Namentlich der Risikoausschuss sei von qualifizierten Experten besetzt. Ein Teilnehmer bohrte nach und wollte wissen, ob sich der Aufsichtsrat gegebenenfalls auch selbst aktiv Informationen aus den einzelnen Institutionen des Unternehmens hole. Krekeler sah auch hier keine Defizite. Man „lasse sich die Leute gegebenenfalls auch kommen“. Ein Mitglied der Gesprächsrunde betonte sodann, dass man heute gene-

*) Wissenschaftlicher Mitarbeiter und Doktorand am Lebrstubl für Bürgerliches Recht, Deutsches und Europäisches Handels-, Gesellschaftsund Wirtschaftsrecht (Professor Dr. Jens Koch), Universität Konstanz rell verschärft auf die Ebenen unterhalb des Vorstands blicken müsse. Auch der Gesetzgeber des KWG müsse sich fragen, ob es zusätzlicher Anforderungen und Mechanismen bedürfe, die gewährleisteten, dass auch untergeordnete Leitungsebenen unmittelbar von den Aufsichtsgremien überwacht werden könnten.

Im Weiteren wurde der Blick auf die konzernweiten Aspekte des Risikomanagements gerichtet. Ein Teilnehmer merkte zunächst an, dass es in der Praxis schwierig sei, eine Momentaufnahme über die bestehenden finanziellen Risiken für den Konzern zu bekommen. Die Rechnungslegung leide vor allem daran, dass viele, insbesondere ausländische Töchter, zu unterschiedlichen Zeiten ihren Abschluss vorlegten. Krekeler räumte gewisse Schwierigkeiten ein. Letztlich sei es im Konzern aber schon möglich, jederzeit einen Überblick über die finanziellen Risiken in den einzelnen Gesellschaften zu bekommen. Sodann wurde herausgearbeitet, dass vor allem das Gesellschaftsrecht einer konzernweiten Risikokontrolle Grenzen setzen könne. Namentlich im faktischen Aktienkonzern stoße die Pflicht, konzernweite Risiken zu kontrollieren, an ihre Grenzen. Ohne die Mitwirkung des Tochtervorstands gehe hier gar nichts. Die Tochtergesellschaften vermittelten aber nicht zuletzt durch die Pflicht zum Verlustausgleich, Haftungszusagen sowie Garantieerklärungen auch im faktischen Aktienkonzern vielmals existenzgefährdende Risiken. Sethe (ZBB 2012, 357, in diesem Heft) sah hier aus der Perspektive der Obergesellschaft indes keine größeren Schwierigkeiten. In der Praxis funktioniere die Zusammenarbeit. Der faktische Konzern habe sich hier bewährt. Das Problem liege vielmehr im Aufsichtsrecht. Hier verfüge man über keine Werkzeuge, um in die Tiefen des Konzerns vorzudringen. Rettungsanker sei allein $\S 35 \mathrm{KWG}$, der es im äußersten Fall erlaube, den Konzern „zuzumachen“. Krekeler ergänzte, dass die Aufsicht in seiner Praxis immer die gewünschten Informationen erhalten habe, auch wenn es um Belange einer Tochtergesellschaft ging. 